10. Yi X, Chan CW, Yeo KJ. Is adding $\operatorname{IgM}$ antibody to polymerase chain reaction testing useful for COVID-19 travel screening? Am J Clin Pathol 2021; 155: 321-3.

11. Iyer AS, Jones FK, Nodoushani A, et al. Persistence and decay of human antibody responses to the receptor binding domain of SARS CoV-2 spike protein in COVID-19 patients. Sci Immunol 2020; 5: eabe0367.

12. Hueston L, Kok J, Guibone A, et al. The antibody response to SARSCoV-2 infection. Open Forum Infect Dis 2020; 7: ofaa387.

DOI: https://doi.org/10.1016/j.pathol.2022.03.001

\section{Vicious cycle of hypertriglyceridaemia and hyperglycaemia in an atypical case of lipoprotein lipase deficiency}

To the Editor,

Severe hypertriglyceridaemia, defined as plasma triglycerides $>10 \mathrm{mmol} / \mathrm{L},{ }^{1}$ is primarily polygenic and contributed by multiple acquired factors including diet, obesity, alcoholism, diabetes mellitus, hypothyroidism, paraproteinaemia and drugs. $^{2}$ On the other hand, familial chylomicronaemia syndrome refers to a group of rare disorders of hypertriglyceridaemia, the most common being lipoprotein lipase deficiency (MIM \#238600) due to pathogenic variants in the $L P L$ gene (MIM *609708), with an estimated prevalence of 1 in 1,000,000. It is an autosomal recessive disorder characterised by impaired plasma clearance of both chylomicrons and very low-density lipoproteins. The majority of patients present before 10 years of age with recurrent pancreatitis secondary to severe hypertriglyceridaemia.

We report an atypical case of lipoprotein lipase deficiency, with the patient presenting in late middle age without any history of pancreatitis or recurrent abdominal pain.

The patient, then a 56-year-old man, was admitted for transient ischaemic attack in July 1999. Fasting blood tests showed triglycerides $28.8 \mathrm{mmol} / \mathrm{L}$, total cholesterol 8.2 $\mathrm{mmol} / \mathrm{L}$ and glucose $6.4 \mathrm{mmol} / \mathrm{L}$. Apart from impaired fasting glycaemia, no other secondary causes of dyslipidaemia were identified. He had no family history of hypertriglyceridaemia. Moderate hypertriglyceridaemia persisted after diet modification and gemfibrozil, which was started in September 1999.

At 62 years of age, he was diagnosed with type 2 diabetes mellitus which was initially managed with diet modification alone. Both his glycaemic control and hypertriglyceridaemia gradually worsened. Metformin and atorvastatin were started at 67 years of age, and gliclazide was added at 72 years of age. Two years later, both his HbA1c (6.1\%) and plasma triglyceride level $(2.3 \mathrm{mmol} / \mathrm{L})$ reached a nadir.

However, his disease control deteriorated afterwards. At 77 years of age, his HbA1c increased to $9.2 \%$ despite increasing dose of metformin and gliclazide, and plasma triglycerides increased to $42.6 \mathrm{mmol} / \mathrm{L}$ despite concurrent use of rosuvastatin, fenofibrate and ezetimibe. Therefore, he was admitted for management of severe hyperlipidaemia. Repeat blood tests showed triglycerides $21.2 \mathrm{mmol} / \mathrm{L}$, total cholesterol $10.6 \mathrm{mmol} / \mathrm{L}$, HDL cholesterol $0.5 \mathrm{mmol} / \mathrm{L}$, direct LDL cholesterol $1.7 \mathrm{mmol} / \mathrm{L}$, apolipoprotein A1 $1.11 \mathrm{~g} / \mathrm{L}$ (reference interval 1.10-1.80 g/L) and apolipoprotein B $1.68 \mathrm{~g} / \mathrm{L}$
(0.49-1.15 g/L). Lipoprotein electrophoresis showed type V hyperlipidaemia with dense chylomicron and VLDL lipoprotein bands. Insulin was started which improved both his glycaemic control and lipid profile. He was discharged a week later. His plasma triglycerides and HbA1c levels are summarised in Fig. 1.

A monogenic cause of hypertriglyceridaemia, in particular lipoprotein lipase deficiency, was suspected because of the disease severity and resistance to treatment. Sanger sequencing of the $L P L$ gene (reference sequence NM_000237.2) revealed apparent compound heterozygosity for two previously reported pathogenic variants, c. $292 \mathrm{G}>\mathrm{A}$ p.(Ala98Thr) (dbSNP rs145657341) and c.835C $>$ G p.(Leu279Val) (dbSNP rs371282890). ${ }^{4,5}$ The diagnosis of lipoprotein lipase deficiency was thus confirmed. Cascade screening for at-risk family members was advised, and they preferred biochemical screening to genetic testing. Further history revealed that the patient was taking fish oil supplements which are contraindicated in lipoprotein lipase deficiency. Those supplements were subsequently stopped.

There is no known genotype-phenotype correlation in lipoprotein lipase deficiency. ${ }^{3}$ The $L P L$ Ala98Thr variant decreases both the secretion and the catalytic activity of lipoprotein lipase, although some residual activity is preserved. On the other hand, the $L P L$ Leu279Val variant almost abolishes the catalytic activity. ${ }^{4}$ Two other patients were reported to have the same compound heterozygous $L P L$ variants as in our patient in the literature. One presented at 28 years of age with acute pancreatitis during late pregnancy, which is physiologically associated with hypertriglyceridaemia, and the other was diagnosed at 58 years of age after three episodes of pancreatitis.

The LPL Ala98Thr and Leu279Val variants have allele frequencies of $33 / 19950(0.17 \%)$ and $31 / 19954(0.16 \%)$ among East Asians in the Genome Aggregation Database v2.1.1. Because the variants are unlinked, ${ }^{4,5}$ this suggests a much higher prevalence of lipoprotein lipase deficiency among East Asians at around 1 in 100,000, which is still an underestimate as other disease-causing variants have not been taken into account. Widespread implementation of genetic testing should help uncover more undiagnosed cases among East Asians.

Surprisingly, our now 78-year-old patient has never suffered from pancreatitis or recurrent abdominal pain. This is unusual as most patients (50-80\%) with lipoprotein lipase deficiency will develop pancreatitis. ${ }^{1}$ Our patient had good past health until 56 years of age when hypertriglyceridaemia was discovered after an episode of transient ischaemic attack.

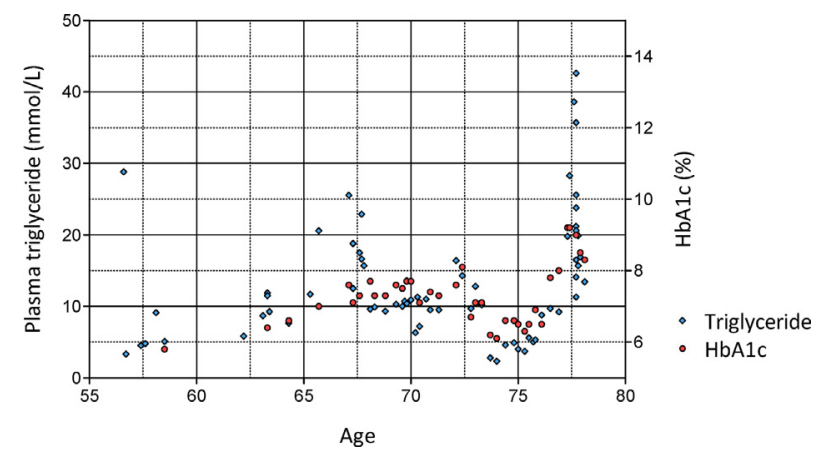

Fig. 1 Plasma triglyceride (mmol/L; left axis) and $\mathrm{HbAlc}$ (\%; right axis) plotted against age of our patient. 
Because of the late diagnosis of hypertriglyceridaemia, he was misclassified as very unlikely to have lipoprotein lipase deficiency based on a recently published scoring system. This highlights the limitations of the scoring system because of the phenotypic variability in this disorder. This unusual presentation in our patient could possibly be contributed by his healthy lifestyle with a fat-restricted diet and a high level of physical activity, which may help to reduce hypertriglyceridaemia.

Lipoprotein lipase deficiency causes impaired hydrolysis of both chylomicron and VLDL triglycerides. In paediatric and adolescent patients with lipoprotein lipase deficiency, type I hyperlipidaemia (isolated elevation in chylomicrons) is the more common phenotype as hepatic lipase may still hydrolyse VLDL triglycerides efficiently. On the contrary, type $\mathrm{V}$ hyperlipidaemia (elevation in both chylomicrons and VLDL) is observed in most adult patients with lipoprotein lipase deficiency. Therefore, type I hyperlipidaemia is considered to be a specific but not sensitive feature for diagnosis of familial chylomicronaemia syndrome. ${ }^{6}$ In patients with type $\mathrm{V}$ hyperlipidaemia, especially if the hypertriglyceridaemia is severe and resistant to conventional treatments, the diagnosis of familial chylomicronaemia syndrome must be considered. In addition, as in our case, the presence of type 2 diabetes mellitus does not rule out the possibility of a concomitant inherited lipid disorder leading to hypertriglyceridaemia.

To the best of our knowledge, this is the first report describing a close correlation between hypertriglyceridaemia and hyperglycaemia in a patient with lipoprotein lipase deficiency for over 20 years (Fig. 2). Lipoprotein lipase deficiency may cause recurrent pancreatitis which may result in pancreatic insufficiency and diabetes mellitus. However, patients may nevertheless develop diabetes mellitus even without pancreatitis. This is because hypertriglyceridaemia is known to induce insulin resistance, and indeed impaired insulin sensitivity has been reported in both carriers and patients with lipoprotein lipase deficiency. ${ }^{7-9}$ Insulin resistance leads to hyperglycaemia, which in turn exacerbates the underlying hypertriglyceridaemia. Therefore, we conclude that a vicious cycle of hypertriglyceridaemia and hyperglycaemia has taken place in our case. Despite the strong genetic predisposition to hypertriglyceridaemia, our patient once had satisfactory disease control at 74 years of age after intensive treatment. The recent deterioration in glycaemic control was

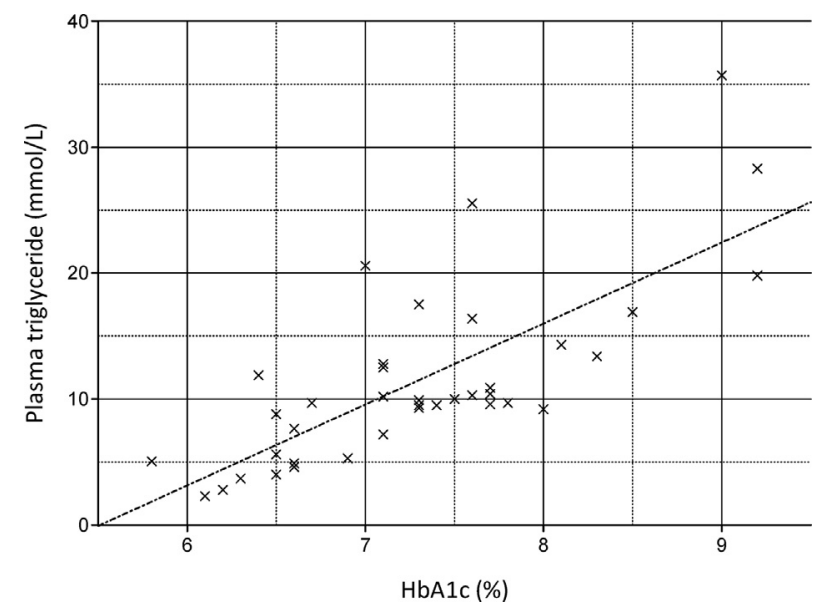

Fig. 2 Plasma triglyceride (mmol/L) plotted against HbA1c (\%) of our patient. probably due to beta-cell failure, which is not uncommon in patients with long-standing type 2 diabetes mellitus. Therefore, for both carriers and patients with lipoprotein lipase deficiency, early diagnosis and intervention of hypertriglyceridaemia are important to prevent the development of type 2 diabetes mellitus. In addition, any diabetes mellitus should be treated intensively to abort the vicious cycle.

Conventional lipid-lowering therapies are not effective in lipoprotein lipase deficiency. Instead, low fat diet (15-20 g/ day) and tight control of other risk factors of hypertriglyceridaemia are essential. Patient history regarding the use of fish oil supplements is important, as these may be used as over-the-counter remedies for dyslipidaemia, but are contraindicated in lipoprotein lipase deficiency as they may worsen hypertriglyceridaemia. ${ }^{3}$ Glybera (alipogene tiparvovec), a gene therapy based on the gain-of-function $L P L$ S447X variant, was once available in Europe for lipoprotein lipase deficiency. However, due to its prohibitive cost and limited efficacy, it failed to gain national reimbursement in any European countries and was withdrawn in $2017 .{ }^{10}$ Other novel therapies are being developed, for example, volanesorsen (an antisense APOC3 inhibitor) and evinacumab (a monoclonal antibody inhibitor of angiopoietin-like protein). These may significantly improve the disease control in the future. $^{11}$

Siblings of the proband have a $25 \%$ chance of having the disease and a 50\% chance of being a carrier, while both parents and offspring of the proband are obligate carriers. Carriers may have normal lipid profile, mixed hyperlipidaemia, isolated hypercholesterolaemia or isolated hypertriglyceridaemia. They may have increased risks of diabetes mellitus and cardiovascular events. Cascade screening should therefore be offered for all at-risk family members. ${ }^{3}$ Biochemical monitoring with lipid profile is recommended for all obligate carriers of lipoprotein lipase deficiency for early diagnosis and intervention as their phenotype could vary with time. ${ }^{12}$

In summary, we report an atypical case of lipoprotein lipase deficiency which presented in late middle age with transient ischaemic attack. The patient later developed type 2 diabetes mellitus, and a close correlation between plasma triglycerides level and HbA1c was observed. Without a history of pancreatitis, the diagnosis was only apparent after a vicious cycle between hypertriglyceridaemia and hyperglycaemia had been established. This case reinforces the understanding that early diagnosis and intensive treatment of both hypertriglyceridaemia and diabetes mellitus (if any) are important in lipoprotein lipase deficiency. History regarding the use of fish oil supplements is important as they are contraindicated in lipoprotein lipase deficiency. Cascade screening is recommended for at-risk family members in order to prevent complications.

Conflicts of interest and sources of funding: The authors state that there are no conflicts of interest to disclose.

\section{Hok-Fung Tong ${ }^{1}$, Matthew Tai-Fai Kwan ${ }^{2, *}$, Kin- Wah Chan', Yeow-Kuan Chong ${ }^{1}$}

${ }^{1}$ Department of Pathology, Princess Margaret Hospital, Hong Kong; ${ }^{2}$ Department of Medicine and Geriatrics, Princess Margaret Hospital, Hong Kong; "Current 
affiliation: Department of Medicine and Geriatrics, Caritas Medical Centre, Hong Kong

Contact Dr Yeow-Kuan Chong.

E-mail: cyk280a@ha.org.hk

1. Garg A, Garg V, Hegele RA, Lewis GF. Practical definitions of severe versus familial hypercholesterolaemia and hypertriglyceridaemia for adult clinical practice. Lancet Diab Endocrinol 2019; 7: 880-6.

2. Dron JS, Wang J, Cao H, et al. Severe hypertriglyceridemia is primarily polygenic. J Clin Lipidol 2019; 13: 80-8.

3. Burnett JR, Hooper AJ, Hegele RA. GeneReviews: familial lipoprotein lipase deficiency. Cited 19 Jan 2021. http://www.ncbi.nlm.nih.gov/ books/NBK1308/

4. Chan LYS, Lam C-W, Mak Y-T, et al. Genotype-phenotype studies of six novel LPL mutations in Chinese patients with hypertriglyceridemia. Hum Mutat 2002; 20: 232-3.

5. Chen T-Z, Xie S-L, Jin R, Huang Z-M. A novel lipoprotein lipase gene missense mutation in Chinese patients with severe hypertriglyceridemia and pancreatitis. Lipids Health Dis 2014; 13: 52.

6. Moulin P, Dufour R, Averna M, et al. Identification and diagnosis of patients with familial chylomicronaemia syndrome (FCS): expert panel recommendations and proposal of an "FCS score". Atherosclerosis 2018; 275: 265-72.

7. Yang T, Pang C-P, Tsang M-W, et al. Pathogenic mutations of the lipoprotein lipase gene in Chinese patients with hypertriglyceridemic type 2 diabetes. Hum Mutat 2003; 21: 453.

8. Mingrone G, Henriksen FL, Greco AV, et al. Triglyceride-induced diabetes associated with familial lipoprotein lipase deficiency. Diabetes 1999; 48: 6

9. Raupp P, Keenan C, Dowman M, Nath R, Hertecant J. Lipoprotein lipase deficiency and transient diabetes mellitus in a neonate. $J$ Inherit Metab Dis 2002; 25: 413-4.

10. Senior M. After Glybera's withdrawal, what's next for gene therapy? Nat Biotechnol 2017; 35: 491-2.

11. Ng DM, Burnett JR, Bell DA, Hegele RA, Hooper AJ. Update on the diagnosis, treatment and management of rare genetic lipid disorders. Pathology 2019; 51: 193-201.

12. Babirak SP, Iverius PH, Fujimoto WY, Brunzell JD. Detection an characterization of the heterozygote state for lipoprotein lipase deficiency. Arteriosclerosis 1989; 9: 326-34.

DOI: https://doi.org/10.1016/j.pathol.2021.07.008

\section{Nuclear magnetic resonance spectroscopy-based urinalysis for a young girl with extreme hypoglycaemia}

\section{To the Editor,}

Hypoglycaemia is not an uncommon medical emergency in paediatric patients. Untreated hypoglycaemia can be rapidly fatal in young vulnerable subjects. Unlike hypoglycaemia in adults, inherited metabolic disorders (IMD) should be considered as one of the key differential diagnoses in paediatric patients and early diagnosis is important to guide appropriate management and prevent further metabolic insult. Here, we report a case of recurrent and severe hypoglycaemia in a 1-year-old girl who was diagnosed with fructose-1,6-bisphosphatase (FBP1) deficiency, a lifethreatening and rare IMD condition which can be effectively managed by lifestyle and dietary modification. We will discuss the clinical use of NMR-based urinalysis in the rapid diagnosis of FBP1 deficiency and describe the use of the glycerol-kinase-based triglycerides assay as a quick approach to quantitate glycerol, a pathognomonic marker for this condition. This study was approved by Institutional Review Board (Reference Number: UW 21-150).

The 1-year-old patient was referred to us for metabolic investigations, presenting with acute and ketotic hypoglycaemia. Before this admission, she had another episode of a similar nature at 11 months old, which was managed as a viral infection in another hospital. The patient had an unremarkable birth history and was born to non-consanguineous parents. A dried blood spot newborn screening test was performed in a private centre with unremarkable findings. She had normal development and growth all along. On admission, she presented with extreme hypoglycaemia (plasma glucose $0.5 \mathrm{mmol} / \mathrm{L}$ ), ketosis [plasma betahydroxyburytate $(\mathrm{BOHB}) 5.5 \mathrm{mmol} / \mathrm{L}$, reference interval (RI) <0.6], lactic acidosis (plasma lactate $10.1 \mathrm{mmol} / \mathrm{L}$, RI 0.7-2.1; pH 7.29; RI 7.35-7.45) and hyperuricaemia (0.7 $\mathrm{mmol} / \mathrm{L}$, RI $0.11-0.3)$. She also had an elevated lactate-topyruvate (L:P) ratio of 42 and an elevated BOHB-toacetoacetate (AcAc) ratio of 8 . On physical examination, the patient appeared irritable and lethargic with decreased tone over four limbs. She had warm peripheries and a satisfactory hydration status. Her pulse was $106 \mathrm{BPM}$, and she was tachypnoeic. There was no significant hepatomegaly. The patient remained arousable during physical examination despite a fluctuating consciousness. There was no known positive family history of hypoglycaemia, IMD or sudden infant death. Her twin brother was otherwise healthy with unremarkable development. The patient was given intravenous dextrose infusion, and her condition gradually improved with no other complications upon discharge.

A urine sample was saved during hypoglycaemic attack in this patient and was sent to our laboratory for further metabolic work-up. Urine organic acid (UOA) analysis with ethyl acetate extraction by gas chromatography-mass spectrometry (GC-MS; 5973N Gas Chromatography system; Agilent, USA) detected marked increases in urinary glycerol, lactate, AcAc and BOHB. Nuclear magnetic resonance (NMR)-based urinalysis ( ${ }^{1} \mathrm{H}-\mathrm{NMR}$; $600 \mathrm{MHz}$ Avance; Bruker, USA) showed hyperexcretion of glycerol, measuring $47.5 \mathrm{mmol} /$ mmol creatinine (97.5th percentile $0.05 \mathrm{mmol} / \mathrm{mmol} \mathrm{Cr}$ ), BOHB $49 \mathrm{mmol} / \mathrm{mmol} \mathrm{Cr}$ (97.5th percentile $0.008 \mathrm{mmol} /$ mmol Cr), AcAc $10 \mathrm{mmol} / \mathrm{mmol} \mathrm{Cr}$ (97.5th percentile not available), lactate $71 \mathrm{mmol} / \mathrm{mmol} \mathrm{Cr}$ (97.5th percentile $<0.116 \mathrm{mmol} / \mathrm{mmol} \mathrm{Cr}$ ) and creatinine $1.2 \mathrm{mmol} / \mathrm{L}$ (Fig. 1).

Clinically, ketotic hypoglycaemia with glyceroluria can be seen in FBP1 or glycerol kinase (GK) deficiency. Since GK deficiency is an X-linked condition, our patient will be a manifested female if she is affected by GK deficiency. She also has an unaffected twin brother and therefore we consider FBP1 deficiency is more likely in our case. Genetic analysis was arranged and we identified a homozygous variant, NM_000507.4:c.959dupG; NP_000498.2:p.Ser321IlefsTer13 in the FBP1 gene and this is a known diseasecausing variant for FBP1 deficiency. This patient had a good clinical response after lifestyle and dietary modifications.

The gluconeogenic pathway and the glycolytic pathway proceed in opposite directions, involving mostly bidirectional enzymes intercalated with key irreversible enzymes unique to each pathway. In particular, FBP1, pyruvate carboxylase, phosphoenolpyruvate carboxykinase and glucose 6- 